FACTORES DETERMINANTES DE LA PERSONALIDAD DE MARCA. UN ESTUDIO EMPÍRICO EN ESCENARIOS VERACRUZANOS

\title{
FACTORES DETERMINANTES DE LA PERSONALIDAD DE MARCA. UN ESTUDIO EMPÍRICO EN ESCENARIOS VERACRUZANOS
}

\section{DETERMINING FACTORS OF BRAND PERSONALITY. AN EMPIRICAL STUDY IN THE STATE OF VERACRUZ}

\author{
Ena Yuritze Barón López* , Arturo García Santillán**
}

* Researcher and Professor in Marketing at Marketing Studies Department Universidad Central BogotáColombia. ORCID: https://orcid.org/0000-0002-2659-450

** Researcher and Coordinator of Doctoral Program in Management Science UCC Business School Universidad Cristóbal Colón. Carr. Veracruz-Medellín s/n Col. Puente Moreno Boca del Río, Veracruz. México. Tel. 01 (229) 9232950 Ext. 6285 C.P. 94271. ORCID: https://orcid.org/0000-0001-7284-5959 Email: agarcias@ucc.mx, arturogarciasantillan@yahoo.com.mx

Dirección para recibir correspondencia: enabaronlopez@gmail.com 
FACTORES DETERMINANTES DE LA PERSONALIDAD DE MARCA. UN ESTUDIO EMPÍRICO EN ESCENARIOS VERACRUZANOS

\section{RESUMEN}

OBJETIVO: Demostrar la relación que puede existir entre los rasgos de la personalidad de marca humana y la personalidad de una marca en un contexto latino.

MATERIAL Y MÉTODO: Los análisis de este estudio se hacen a partir de la propuesta de Jenifer Aaker y la escala de personalidad humana que se consideran todavía de gran importancia para poder explicar esta asociación de valores.

RESULTADOS: Los datos se recogieron en el contexto latino, específicamente en el estado de Veracruz en México esto con el propósito de confirmar si la escala tiene la misma fiabilidad en contextos diferentes al original. Mediante un análisis factorial se buscó aceptar las hipótesis de propuestas.

CONCLUSIONES: Este estudio también puede explicar que si existe una personalidad de marca relacionadas con las marcas de botanas o snacks, sin embargo es importante que en futuros trabajos se pueda comprobar si esta relación puede influenciar la preferencia del consumidor.

KEYWORDS: Personalidad de marca, snacks, botanas, contexto latino.

\section{ABSTRACT}

OBJECTIVE: To demonstrate the relation between personality traits and personal branding in a latin context.

MATERIAL AND METHOD: The analysis of this study is based on Jenifer Aaker's proposal and the personality scale still considered main reference in order to explain the association of these values.

RESULTS: The data was collected in a latin context, especifically in the state of Veracruz in Mexico, with the purpose of confirming if the scale ha the same reliability in other contexts. Hypotheses confirmation were validated through a factorial analysis. 
FACTORES DETERMINANTES DE LA PERSONALIDAD DE MARCA. UN ESTUDIO EMPÍRICO EN ESCENARIOS VERACRUZANOS

CONCLUSIONS: This study can also explain the existence of personal branding with products as snacks, however it is important to consider this relation in future works and if it can create influence in consumers.

KEY WORDS: Personal Branding. Snacks. Latin context.

\section{INTRODUCCIÓN}

En el marketing de consumo, las marcas proporcionan a menudo los criterios principales de diferenciación entre ofertas de la competencia, y como tales, pueden ser fundamentales para el éxito de las empresas (Wood L, 2000). Al respecto de esto, la asociación Americana de Marketing define que una marca es un "nombre, termino, diseño, símbolo o alguna característica que identifique a un vendedor de bienes o servicios, que lo distinga frente a otros competidores. Comprender y conocer el contenido y la estructura de una marca es importante porque eso, tiene una influencia en lo que viene a la mente del consumidor cuando piensa acerca de una marca, por ejemplo en respuesta a las actividades de marketing de esa marca (Kendall 1993). Asimismo, Larios, Estrada y Luna (2015, p. 123), argumentan que cuando "se considera la función de la marca de un producto, desde la perspectiva del consumidor, se reconocen las mismas ventajas que ésta supone para una empresa, conceptos como el valor de la marca, o conjunto de activos y pasivos vinculados a la misma, su nombre y símbolo, que incorporan o disminuyen el valor suministrado por un bien, servicio, idea o experiencia". El concepto y la realidad de las relaciones de marca, ayudan a los gerentes a desarrollar y posicionar estrategias y tácticas de marketing dirigidas a reforzar la intensidad de las relaciones entre consumidores y marca (Ambroise et al, 2004). De ahí que, en el dominio del marketing, la marca podría ser el elemento más importante para diferenciarse de la competencia; en consecuencia es fundamental estudiar herramientas que permitan analizar las características de las marcas y como ellas influyen en la decisión de compra del consumidor, entonces las estrategias de marketing buscan herramientas que optimicen el trabajo de posicionamiento de las marcas en la mente y los corazones de los consumidores, (Barón \& Bautista, 2018) comentan que apoyados en esta observación, los creadores de marca han pensado en transferir aquellos rasgos humanos a las marcas de allí que se busque formas en las que los consumidores se apropien de las marcas y se identifiquen con ellas. 
FACTORES DETERMINANTES DE LA PERSONALIDAD DE MARCA. UN ESTUDIO EMPÍRICO EN ESCENARIOS VERACRUZANOS

Después de Aaker, hay otros autores que han desarrollado propuestas para medir los rasgos de la personalidad de una marca; la aproximación "Lexical" (Caprara, Barbaranelli, Guido, 1997, 2001). Asimismo, Allen y Olson (1995), proponen una escala de tipo narrativo, y en la misma idea, para contribuir a los estudios y refinar la capacidad predictiva de las escalas, Ambroise et al (2004) proponen una escala para medir la personalidad de una marca, la cual, a su vez tiene la capacidad de predecir su elección. En este estudio, se retoma la escala propuesta por Aaker (1997), teniendo en cuenta que es una escala altamente reconocida en el ámbito académico y posee altos valores de fiabilidad, en el estudio de Aaker el Alpha de Cronbach arrojó una consistencia interna para cada una de las dimensiones de acuerdo a lo siguiente: sinceridad $=.93$, emoción $=.95$, competencia $=.93$, sofisticación $=.91$ y rudeza $=.90$. En concordancia con lo que sugiere Ambroise "un fuerte trabajo es aún necesario en el desarrollo de términos, definición de conceptos y escalas de medida de la personalidad de marca y sobre todo la aplicación de estas herramientas en diferentes culturas", como pudiera ser en el contexto latinoamericano (Ambroise et al, 2004, p. 3).

Con el fin de hacer nuevos aportes a los estudios relacionados con la personalidad de marca y considerando la importancia que esto puede traer a las futuras decisiones estratégicas en el marketing, se busca responder al siguiente cuestionamiento ¿Cuáles rasgos de la personalidad representan de manera más significativa las marcas de snacks en un contexto Latinoamericano?; es decir, ¿Cuál es la estructura de variables latentes que mejor describe la personalidad de marca de productos snacks en un contexto latino?. Para poder responder a la pregunta central del estudio, es necesario llevar a cabo la aplicación del instrumento para la recogida de datos para el estudio empírico, en un contexto latino, por lo que el estudio se realiza en la ciudad de Veracruz, México, de modo que se podrá verificar la estabilidad del instrumento en un contexto diferente al original, lo que favorecerá en el esfuerzo por intentar confirmar nuevos hallazgos.

Para responder a la pregunta de investigación y logar el objetivo del estudio, se replica el modelo de Aaker (1997), el cual integra las dimensiones que definen una estructura de variables que explican la personalidad de marca (ver figura 1). 
FACTORES DETERMINANTES DE LA PERSONALIDAD DE MARCA. UN ESTUDIO EMPÍRICO EN ESCENARIOS VERACRUZANOS

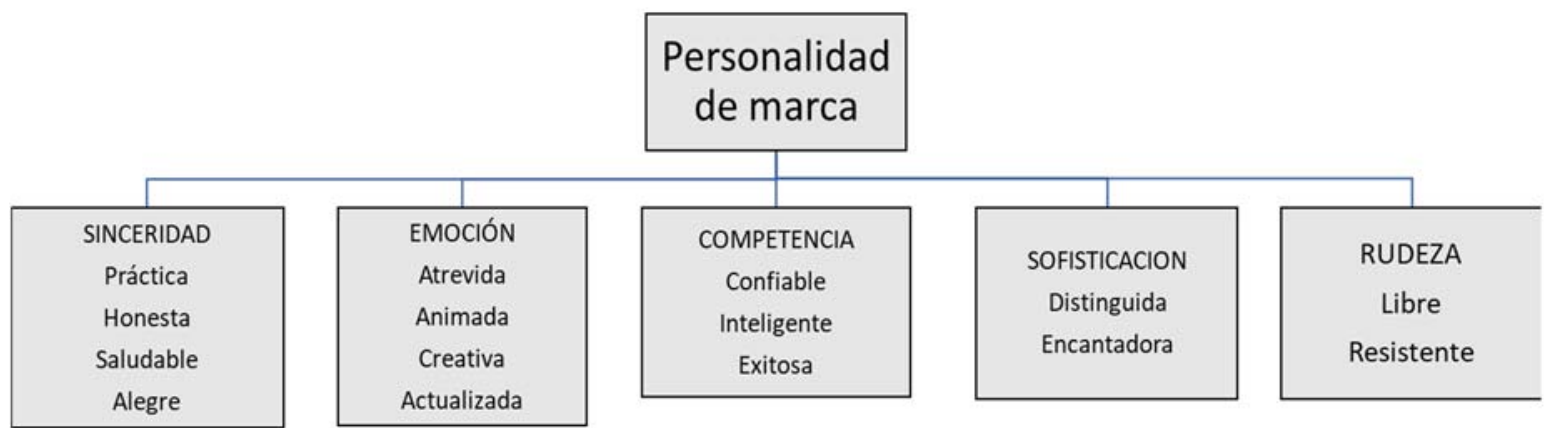

Figura 1. Personalidad de marca.

Fuente: Constructo de la personalidad de marca propuesta por Aaker (1997).

En la revisión especializada a este constructo, Azoulay y Kapferer (2003) reflexionan sobre el concepto sobre personalidad de marca y refieren que "la personalidad de la marca es el conjunto de rasgos de la personalidad humana que son a la vez aplicables y relevantes para las marcas". Para Aaker, la personalidad de la marca es un "set de características humanas que son asociadas a la marca". Continúa señalando, que los consumidores hacen una asociación directa entre los rasgos de la personalidad y la marca; de la misma forma, las marcas construyen y constituyen un imaginario que se define como un "set de características asociadas con el típico usuario de una marca" (Aaker, 1997). Desde esta perspectiva, los rasgos de la personalidad de los consumidores asociados con una marca son transferidos directamente sobre la marca (McCracKen, 1989).

Desde el punto de vista de la psicología, se exploran las emociones para la creación o consolidación de la personalidad de marcas, porque el mundo contemporáneo exige, cada vez más, reconocer las emociones dentro de los ámbitos culturales existentes y cambiantes (Barón \& Bautista, 2018); es decir, que las marcas deben hacer una relación coherente con las significaciones culturales para que el sistema de valores entre marcas y consumidores sea estratégico y eficaz para el posicionamiento. Varios autores han considerado el estudiar la marca desde el referente de la personalidad humana, a saber, en la década de los ochenta las investigaciones sobre personalidad de marca estaban enfocadas en la influencia del autoconcepto; estos trabajos proponen que el auto concepto del consumidor influye en la evaluación de las marcas y su compra (Sirgy, 1982; Belk, 1988; Malhotra, 1988).

Más adelante, Zinkhan, Haytko y Ward (1996), estudian la teoría del auto concepto en los resultados de la efectividad de la publicidad;eEllos desarrollan dos aproximaciones, una que va 
FACTORES DETERMINANTES DE LA PERSONALIDAD DE MARCA. UN ESTUDIO EMPÍRICO EN ESCENARIOS VERACRUZANOS

desde lo académico (teórico) y la otra desde la perspectiva gerencial a partir de entrevistas a expertos de la publicidad.

El propósito es acercar ambos enfoques al entendimiento del fenómeno del auto concepto. Posteriormente, en Aaker (1997) propone una escala para medir la personalidad de marca a partir de la investigación sobre los cinco grandes componentes de la personalidad humana (Norman, 1963).

\section{Escalas de medida de la personalidad de marca.}

La escala de personalidad de marca es considerada una herramienta que mide el conjunto de características humanas, la cual se relacionan con una marca; es decir, es un "set de características asociadas con el típico usuario de una marca" (Aaker, 1997). Sin duda, los trabajo seminales desarrollados por Aaker (1997), son los más citados en el campo de la personalidad de marca. En sus estudios, identificó cinco posibles dimensiones de la personalidad de marcas o sets de atributos humanos asociados particularmente con marcas consumidas en Norte América. Las dimensiones incluidas son: Sinceridad, emoción, capacidad, sofisticación y robustez (Dall'Olmo, 2009). Aaker propone la primera escala de medida de la personalidad de una marca con respecto a los rasgos de la personalidad de un individuo. La propuesta conceptual, teórica y práctica desarrollada por Aaker se halla esencialmente soportada en la escala de medición de personalidad humana llamada "Big Five", que hasta donde se conoce en este artículo, es la que más se ajusta a una descripción de los rasgos de la personalidad humana (Allport, 1937; Cattell, 1950 y Eysenck, 1960).

En la misma idea sobre las escalas de personalidad de marca, otros autores han desarrollado instrumentos sustentados en los estudios seminales de Aaker, ejemplo de ello, Caprara, Barbaranelli y Guido (2001) trabajaron una aproximación lexical para identificar los principales atributos de la personalidad de una marca. Desde esta aproximación, proponen la hipótesis de que los principales rasgos de la personalidad de la marca caracterizan también a los productos. Por su parte, Allen y Olson (1995) ponen en marcha una aproximación narrativa para comprender los antecedentes y las consecuencias de la personalidad de marca. También en Francia, en el estudio de Ferrandi, Valette-Florence (2002) trabajaron la aproximación de la personalidad humana a las marcas desde los trabajos de Aaker y las investigaciones desarrolladas en los mini-markers de Saucier (1994). 
FACTORES DETERMINANTES DE LA PERSONALIDAD DE MARCA. UN ESTUDIO EMPÍRICO EN ESCENARIOS VERACRUZANOS

Es a partir de estos elementos expuestos en el planteamiento inicial, los cuales fueron fundamentados con la revisión a la literatura especializada en el tema de la personalidad de marca, que en este estudio se replica la escala de Aaker, con el objetivo de comprobar la fiabilidad de la escala en un contexto distinto del original. Asimismo, Aaker ha señalado que con esta escala se pueden distinguir los diferentes tipos de personalidad, y las múltiples formas en que las construcciones simbólicas de marcas pueden influenciar las preferencias del consumidor (Aaker, 1997).

P1. La primera proposición entonces de este trabajo está relacionada con que existe una personalidad de marca que corresponde a las marcas de botanas o snacks y que esas pueden influenciar las preferencias del consumidor. La siguiente proposición se relaciona entonces con las construcciones simbólicas de las marcas, la favorabilidad en la asociación de marca difiere de acuerdo en cómo estas son evaluadas. El éxito de los programas de marketing se refleja en la creación de esas asociaciones favorables con la marca, los consumidores creen que la marca tiene atributos y beneficios eso satisface sus necesidades y deseos de tal manera que se forma una actitud positiva general (McCracken 1989)

P2. Existe una asociación favorable entre la marca y la personalidad de la misma. Es a partir de la revisión teórica y empírica a los fundamentos, que dan el soporte a este estudio, que se ratifica el constructo teórico propuesto por Aaker (1997) bajo el cual se aborda el estudio.

Además la pregunta y objetivo de investigación se replantean en los siguientes términos: ¿Cuál es la estructura de variables latentes que mejor describe la personalidad de marca hacia los productos snacks en estudiantes dentro de un contexto latino? Así, para responder a la pregunta se fija como objetivo: identificar el conjunto de variables que determinan la personalidad de marca hacia los productos snacks en estudiantes de un contexto latino.

Además, se proponen las siguientes hipótesis:

$\mathrm{H}_{\mathrm{o}}$ : No existe una estructura de variables latentes que describan la personalidad de marca hacia los productos snacks en estudiantes dentro de un contexto latino.

$\mathrm{H}_{\mathrm{a}}$ : Existe una estructura de variables latentes que describan la personalidad de marca hacia los productos snacks en estudiantes dentro de un contexto latino. 
FACTORES DETERMINANTES DE LA PERSONALIDAD DE MARCA. UN ESTUDIO EMPÍRICO EN ESCENARIOS VERACRUZANOS

De manera específica: $\mathrm{H}_{\mathrm{i}}$ : La personalidad de marca hacia los productos snacks que tienen los alumnos puede ser explicada al menos por un factor.

\section{MATERIAL Y MÉTODO}

La investigación se aborda desde el paradigma cuantitativo. Lo anterior, para responder a la pregunta de investigación, la contracción de las hipótesis y al logro de los objetivos. Su diseño es no experimental dado que no se busca manipular las variables independientes $(X)$, de corte transversal, teniendo en cuenta que la obtención de datos se da sólo una vez durante un tiempo determinado. Para fines de esta investigación la muestra es no probabilística porque la elección de los elementos no depende de la probabilidad, sino de causas relacionadas con las características de la investigación. La muestra de estudio se conformó de 124 personas, habitantes de la ciudad de Veracruz, 75\% personas entre 15 y 25 años, $16 \%$ personas entre 26 y 35 años y el $9 \%$ restante de más de 35 años.

Los criterios de selección de la muestra, consistieron en incluir personas habitantes del estado de Veracruz México y de manera específica: Personas de nacionalidad mexicana, personas que conozcan la marca de Barcel y Sabritas, personas que respondieron la totalidad de la encuesta.

Para la investigación de campo se utiliza la "Escala de personalidad de marca de Aaker" (1997). Este instrumento mide cinco factores: Sinceridad, emoción, competencia, sofisticación y rudeza. Esta escala contiene 15 ítems, donde cada uno representa un rasgo de personalidad de marca asociado a cada una de las dimensiones referidas previamente. El rango de escala va desde 1 (totalmente en desacuerdo) a 5 (totalmente de acuerdo). Para el procesamiento de los datos, captura y posterior análisis de la información se utiliza el programa SPSS v 23. Además, en tabla 1 y 2 se describe cada uno de los indicadores, su concepto, su codificación y las dimensiones con los ítems. 
FACTORES DETERMINANTES DE LA PERSONALIDAD DE MARCA. UN ESTUDIO EMPÍRICO EN ESCENARIOS VERACRUZANOS

Tabla 1

Descripción y codificación de los constructos (Escala personalidad de Marca)

\begin{tabular}{llcl}
\hline Constructo & Conceptualización & Codificación & Ítems \\
\hline Sinceridad & La marca refleja honestidad. & $\mathrm{X}_{1}$ & $1,2,3,4$ \\
Emoción & La marca refleja energía y se asocia con lo joven. & $\mathrm{X}_{2}$ & $5,6,7,8$, \\
Competencia & La marca refleja ser competente e inteligente. & $\mathrm{X}_{3}$ & $9,10,11$ \\
Sofisticación & La marca refleja distinción. & $\mathrm{X}_{4}$ & 12,13 \\
Rudeza & la marca refleja independencia y fortaleza & $\mathrm{X}_{5}$ & 14,15 \\
\hline
\end{tabular}

Fuente: Elaboración propia.

Para la contratación de las hipótesis que guían el estudio, se siguió el procedimiento estadístico propuesto por García-Santillán, Venegas-Martínez, Escalera-Chávez y Córdova-Rangel, (2013), referente al análisis factorial exploratorio con extracción de componentes, porque es de interés en esta investigación, analizar la interrelación entre un grupo de variables y explicarlas en términos de sus dimensiones (Hair, Anderson, Tatham, \& Black, 1999). En una primera instancia para reducir el grupo de factores sujetos a estudio, se sigue el procedimiento (AFE) el cual consiste en lo siguiente:

a) Para validar la pertinencia de la técnica, primeramente se calcula el test de esfericidad de Bartlett con $\mathrm{KMO}$, la prueba de bondad de ajuste $\mathrm{X}^{2}$ con $n \mathrm{gl}$, sig. $=0.05$

b) En un siguiente momento, calcular la matriz anti-imagen para obtener los valores de adecuación muestral por variable (MSA), los cuales en teoría corren a la unidad, de ahí que valores inferiores a 0.5 deberán ser excluidos.

c) Posterior a ello, calcular la matriz de correlaciones para identificar el valor del determinante, el cual en teoría debe ser cercano a 0.00 , considerando que valores cercanos a cero dan evidencia de correlaciones altas, caso contrario, si el valor del determinante es cercano a uno o distante de cero, las correlaciones serán bajas.

d) Bajo el criterio de valor propio, calcular los pesos factoriales y sus comunalidades que permitan obtener el EIGENVALUE (EGV) y con ello, el porcentaje de la varianza explicada.

Finalmente, se establece la región de aceptación o rechazo de las hipótesis de prueba: Rechazar $\mathrm{H}_{\mathrm{o}}$ si $X^{2}$ calculado $>X^{2}$ teórica. 
FACTORES DETERMINANTES DE LA PERSONALIDAD DE MARCA. UN ESTUDIO EMPÍRICO EN ESCENARIOS VERACRUZANOS

\section{RESULTADOS}

El test de esfericidad de Bartlett con $\mathrm{KMO}$, mide la correlación parcial de los constructos, de acuerdo con la teoría cuando el índice $\mathrm{KMO} \geq 0,75$ indica una buena correlación. En la medición de la marca Barcel, existe una correlación parcial significativa, ya que es mayor a 0,75. En el caso de la marca Sabritas, el índice KMO muestra una buena correlación; sin embargo, en los constructos emoción y competencia es menor a 0,8. En términos generales, la bondad de ajuste en los constructos de la escala de Aaker para ambas marcas es bueno ya que es superior a 0,75 .

La matriz anti-imagen muestra los valores de adecuación muestral por variable (MSA), los cuales en teoría corren a la unidad, también se considera que los valores inferiores a 0.5 deberán ser excluidos. Para la marca Barcel, la adecuación muestral de las variables del constructo rudeza y sofisticación no demuestran ser superiores a 0,5 lo que indica que no hay una correlación entre ambos. Las demás variables tienen una buena adecuación muestral de las variables de los demás constructos; en el caso de Sabritas, la adecuación muestral para en el caso de sinceridad y sofisticación no es suficiente para considerarlos, como tampoco en el caso de emoción y sofisticación.

Referente a la marca Barcel dado a todos los componentes superan el 0,5 lo que indica que más del $50 \%$ de la varianza explican la personalidad de la marca; sin embargo, son los componentes con sinceridad, emoción y competencia los que mejor se relacionan dado que las comunalidades de los componentes de los mismos son los que cuentan con un índice más bajo.

Para el caso de la marca Sabritas, los valores de los componentes están cercanos a uno lo que quiere decir que la varianza de ese factor explica las variables que lo componen; sin embargo, los componentes sofisticación y rudeza, son los más alejados del uno, lo que quiere decir que estos no se explican con la misma fuerza la personalidad de la marca en los rasgos que la componen y eso se evidencia en las comunalidades, dado que la comunalidad está explicada como los factores comunes que explican la proporción de la varianza en una variable y teniendo en cuenta que las varianza compartida entre las variables del factor sofisticación es menos cercana a 1 este componente es el que menos explicaría la personalidad de la marca, por el contrario la marca se considera altamente competente, sincera y emocionante. 
FACTORES DETERMINANTES DE LA PERSONALIDAD DE MARCA. UN ESTUDIO EMPÍRICO EN ESCENARIOS VERACRUZANOS

Dado lo anterior y en referencia a las hipótesis planteadas, se puede decir que se aprueba la hipótesis alterna; es decir, que la escala de Aaker de personalidad de marca, es una estructura de variables latentes que describen la personalidad de marca hacia los productos snacks en estudiantes dentro de un contexto latino. Los componentes tienen una alta relación con la personalidad de la marca, pero es importante aclarar que los componentes de sofisticación y rudeza son los que menos se relacionan con la marca.

\section{CONCLUSIONES}

Los resultados encontrados después de los análisis se pueden comprobar que la escala de Aaker puede medir con fiabilidad los rasgos de la personalidad de la marca y en definitiva los consumidores pueden hacer una relación de los rasgos de la personalidad humana con los las marcas.

Este estudio, también puede explicar que si existe una personalidad de marca relacionadas con las marcas de botanas o snacks; sin embargo, es importante que en futuros trabajos se pueda comprobar si esta relación puede influenciar la preferencia del consumidor. De otra parte, a pesar de que los estudios seminales de Aaker se establecieron desde las percepciones de las marcas en Estados Unidos la escala arroja resultados claros sobre la percepción de marcas en contextos latinos es decir que puede considerarse la proposición sobre la asociación favorable entre la marca y la personalidad de la misma.

Pero es importante desarrollar estudios en donde se analicen estos conceptos desde escalas ad-hoc para contextos diferentes, también se considera que a futuro se hagan análisis triangulados que puedan confirmar los resultados de personalidad en las marcas.

\section{REFERENCIAS BIBLIOGRÁFICAS}

Aaker, J. (1997). Dimensions of brand personality. Journal of marketing research, pp. 347-356.

Allen, D. y Olson, J. (1995). Conceptualizing and creating brand personality: A narrative theory approach. Advances in Consumer Research, 22(1), pp. 392-393.

Allport, G. (1937). Personality: A Psychological Interpretation, New York: Holt. 
FACTORES DETERMINANTES DE LA PERSONALIDAD DE MARCA. UN ESTUDIO EMPÍRICO EN ESCENARIOS VERACRUZANOS

Ambroise, L. Ferrandi, J. Merunka, D. y Valette, P. (2004). How well does brand personality predict brand choice? In Asia Pacific Advances in consumer research (2004) pp. 6, 30-38.

American Marketing Association, (1995). American Marketing Association dictionary, 18 de abril de 2016, Recuperado de:

https://www.ama.org/resources/Pages/Dictionary.aspx?dLetter=B

Azoulay, A. y Kapferer, J. (2003). Do brand personality scales really measure brand personality? The Journal of Brand Management, 11(2), pp.143-155.

Barón, E. y Bautista, D. (2018). Personalidad de marca: un estudio de la influencia de la cultura en la formación de rasgo de personalidad en las marcas. In Reflexiones mercadológicas: artesanías, posicionamiento, marca y producto. Bogotá: Universidad Central.

Barón, E. y Bautista, D. (2018). Personalidad de marca: un estudio de la influencia de la cultura en la formación de rasgo de personalidad en las marcas. Recuperado de: en A. W.A. E. Barón, y F. Bautista D. y Martínez, Artesanías, posicionamiento, marca y producto, pp. 7390. Bogotá: Universidad Central.

Belk, R. (1988). Possessions and self. John Wiley y Sons, Ltd. Caprara, G., Barbaranelli, C. y Guido, G. (1998). Personality as metaphor: Extension of the psycholexical hypothesis and the five factor model to brand and product personality description. European advances in consumer research. 3, pp. 61-69.

Caprara, G. Barbaranelli, C., y Guido, G. (2001). Brand personality: how to make the metaphor fit?.Journal of economic psychology, 22(3), pp. 377-395.

Cattell, R. B. (1950). Personality: A systematic theoretical and factual study.

Dall'Olmo Riley. (2009). Â Editor's introduction: brand management. Sage Publications Ltd.

Chernatony, L. y Dall'Olmo Riley, F. (1998). Defining a "brand": Beyond the literature with experts' interpretations. Journal of Marketing Management, 14(5), pp. 417-443.

Eysenck J. (1960). The Structure of Human Personality, London: Methuen.

Ferrandi, J, y Valette-Florence, P. (2002). Premiers test et validation de la transposition d'une échelle de personnalité humaine aux marques. Recherche et Applications en Marketing, 17(3), pp. 21-40.

García-Santillán, A.; Venegas-Martínez, F.; Escalera-Chávez, M.; And Córdova-Rangel, A. (2013) Attitude towards statistics in engineering college: An empirical study in public 
FACTORES DETERMINANTES DE LA PERSONALIDAD DE MARCA. UN ESTUDIO EMPÍRICO EN ESCENARIOS VERACRUZANOS

university (UPA). Journal of Statistical and Econometric Methods 2 Issue 1-3 March (4360)

Hair, J., Anderson, R., Tatham, R. y Black, W. (1999). Multivariate data analysis, fifth edition. Spain: Prentice Hall.

Keller, K. (1993). Conceptualizing, measuring, and managing customer-based brand equity. The Journal of Marketing, 1-22.

Larios, E., Estrada, S. y Luna, E. (2015). La marca ciudad Celaya como estrategia de inserción al city marketing: diagnóstico preliminar. En Contreras, R., Ruiz, H. y Molina R. (coord.). Ejes de crítica y reflexión en torno a la cultura y al desarrollo. México: Pearson.

Malhotra, N. (1981). A scale to measure self-concepts, person concepts, and product concepts. Journal of marketing research, pp. 456-464.

Malhotra, N. (1988). Self-concept and product choice: An integrated perspective. Journal of Economic Psychology, 9(1), pp. 1-28.

McCracken, G. (1989). Who is the celebrity endorser? Cultural foundations of the endorsement process. Journal of consumer research, 3 pp. 10-321.

McCrae, R. y Costa Jr. P. (1999). A five-factor theory of personality. Handbook of personality: Theory and research, 2, pp. 139-153.

McCrae, R. y Costa, P. (1989). The structure of interpersonal traits: Wiggins's circumplex and the five-factor model. Journal of personality and social psychology, 56(4), 586.

Norman, W. (1963). Toward an adequate taxonomy of personality attributes: Replicated factor structure in peer nomination personality ratings. The Journal of Abnormal and Social Psychology, 66(6), 574.

Saucier, G. (1994). Mini-markers: A brief version of goldberg's unipolar Big-Five markers. Journal of personality assessment, 63(3), 506-516. Sirgy, M, (1982). Selfconcept in consumer behavior: A critical review. Journal of consumer research, pp. 287300.

Wood, L. (2000). Brands and brand equity: definition and. Management decision, 38(9), pp. 662669.

Zinkhan, G. Haytko, D. y Ward, A. (1996). Self-concept theory: applications in advertising. Journal of Marketing Communications, 2(1), pp 1-19. 